This item was submitted to Loughborough's Research Repository by the author.

Items in Figshare are protected by copyright, with all rights reserved, unless otherwise indicated.

\title{
Absorbing boundary layers for spin wave micromagnetics
}

\section{PLEASE CITE THE PUBLISHED VERSION}

https://doi.org/10.1016/j.jmmm.2017.06.057

\section{PUBLISHER}

(c) Elsevier

\section{VERSION}

AM (Accepted Manuscript)

\section{PUBLISHER STATEMENT}

This work is made available according to the conditions of the Creative Commons Attribution-NonCommercialNoDerivatives 4.0 International (CC BY-NC-ND 4.0) licence. Full details of this licence are available at: https://creativecommons.org/licenses/by-nc-nd/4.0/

\section{LICENCE}

CC BY-NC-ND 4.0

\section{REPOSITORY RECORD}

Venkat, Guru, H. Fangohr, and A. Prabhakar. 2019. "Absorbing Boundary Layers for Spin Wave Micromagnetics". figshare. https://hdl.handle.net/2134/37322. 


\title{
Absorbing boundary layers for spin wave micromagnetics
}

\author{
G. Venkat ${ }^{\mathrm{a}, *}$, H. Fangohr ${ }^{\mathrm{b}, \mathrm{c}}$, A. Prabhakar ${ }^{\mathrm{a}}$ \\ ${ }^{a}$ Dept. of Electrical Engineering, Indian Institute of Technology Madras, India 600036 \\ ${ }^{b}$ Faculty of Engineering and the Environment, University of Southampton, UK. \\ ${ }^{c}$ European XFEL GmbH, Holzkoppel 4, 22869 Schenefeld, Germany
}

\begin{abstract}
Micromagnetic simulations are used to investigate the effects of different absorbing boundary layers (ABLs) on spin waves (SWs) reflected from the edges of a magnetic nano-structure. We define the conditions that a suitable ABL must fulfill and compare the performance of abrupt, linear, polynomial and tan hyperbolic damping profiles in the ABL. We first consider normal incidence in a permalloy stripe and propose a transmission line model to quantify reflections and calculate the loss introduced into the stripe due to the ABL. We find that a parabolic damping profile absorbs the SW energy efficiently and has a low reflection coefficient, thus performing much better than the commonly used abrupt damping profile. We then investigated SWs that are obliquely incident at $26.6^{\circ}, 45^{\circ}$ and $63.4^{\circ}$ on the edge of a yttrium-iron-garnet film. The parabolic damping profile again performs efficiently by showing a high SW energy transfer to the ABL and a low reflected SW amplitude.
\end{abstract}

Keywords: Magnetization dynamics, micromagnetic simulations, magnonics, spin waves

\section{Introduction}

Easier access to computational resources over the last decade has led to the development of many micromagnetic packages that solve the Landau-Lifshitz (LL) equation for magnetic nano-structures. These packages are being used to study spin wave mode profiles and spectra in a quest to build devices with novel functionalities [1-3]. One approach to these studies is to perturb the ground state with a broadband excitation, and then extract the spin wave (SW) dispersion characteristics [47]. However, simulation boundaries are known to affect the dissipative dynamics of the magnonic spectra in such studies $[8,9]$, and we artificially increase the damping $\alpha$ at the boundaries, to absorb the SW reflections. The increase in $\alpha$ can be smooth, e.g. using a hyperbolic tangent function [10], or abrupt [11]. The latter approach was used to attenuate SW reflections, and to calculate the dispersion and scattering parameters in magnonic devices $[12,13]$. More recently, an exponential increase in damping was used to curb reflections in the study of skyrmions and the Dzyaloshinskii-Moriya interaction in magnetic nanostripes [14, 15].

In this article, we define the return loss using transmission line models, to study the impact of using artificial regions of high $\alpha$, or absorbing boundary layers (ABLs), at the edges of the device. We propose a parabolic increase in $\alpha$ and show that it causes less spurious SW reflections than an abrupt increase in

\footnotetext{
* Corresponding author

Email address: guruvenkat7@gmail.com (G. Venkat)

ABL - Absorbing Boundary Layer; SW - Spin Wave; LL - Landau-Lifshitz; PML - Perfectly Matched Layer; FDTD - Finite Difference Time Domain; GPU - Graphics Processing Unit; FD - Finite Difference; YIG - Yttrium Iron Garnet; Transmission line - Tx line
}

$\alpha$. We compare the parabolic profile against the abrupt, linear and the tan hyperbolic profile, for different angles of incidence. The parabolic profile also aligns the micromagnetic community more closely with the accepted polynomial form of perfectly matched layers (PMLs) in finite difference time domain (FDTD) simulations of Maxwell's equations [16].

To our knowledge, this is the first exhaustive study of ABLs using the graphics processing unit (GPU) accelerated finite difference (FD) micromagnetic package MuMax3 [17]. We also provide the codes for post processing the simulation data and raw data for the figures in a code repository for easy reproduction [18].

\section{Normal incidence of spin waves}

The time evolution of the magnetization is described by the LL equation $[19,20]$

$$
\frac{\partial \mathbf{m}}{\partial t}=\gamma^{\prime}[(\mathbf{m} \times \mathbf{H})+\alpha(\mathbf{m} \times(\mathbf{m} \times \mathbf{H}))],
$$

where $\mathbf{m}=\mathbf{M} / M_{\mathbf{S}}$ is the normalized magnetization, and $\mathbf{M}$ and $\mathbf{H}$ are the total magnetization and effective field at time $t$, respectively. $\gamma^{\prime}=\gamma \mu_{0} /\left(1+\alpha^{2}\right)$, with $\gamma<0$ being the electron gyromagnetic ratio, $\alpha$ the phenomenological damping coefficient and $\mu_{0}$ the permeability of free space. We consider a stripe of permalloy $\left(\mathrm{Ni}_{80} \mathrm{Fe}_{20}\right)$ having dimensions $4000 \times 1000 \times 5 \mathrm{~nm}^{3}$, as shown in Figure 1 (a). The structure was proposed as a micromagnetic sample problem for studying SW dynamics and dispersion [7]. We choose a simple geometry with known solutions for the mode profiles.

The material parameters used for permalloy were the saturation magnetization $M_{\mathrm{s}}=800 \mathrm{kA} / \mathrm{m}$ and exchange constant 
$A=13 \times 10^{-12} \mathrm{~J} / \mathrm{m}$ [7]. No crystalline anisotropy was considered. The cell size was taken as $4 \times 4 \times 5 \mathrm{~nm}^{3}$, such that the cell dimensions are less than the exchange length for permalloy, $l_{\mathrm{ex}} \simeq 5.7 \mathrm{~nm}$.

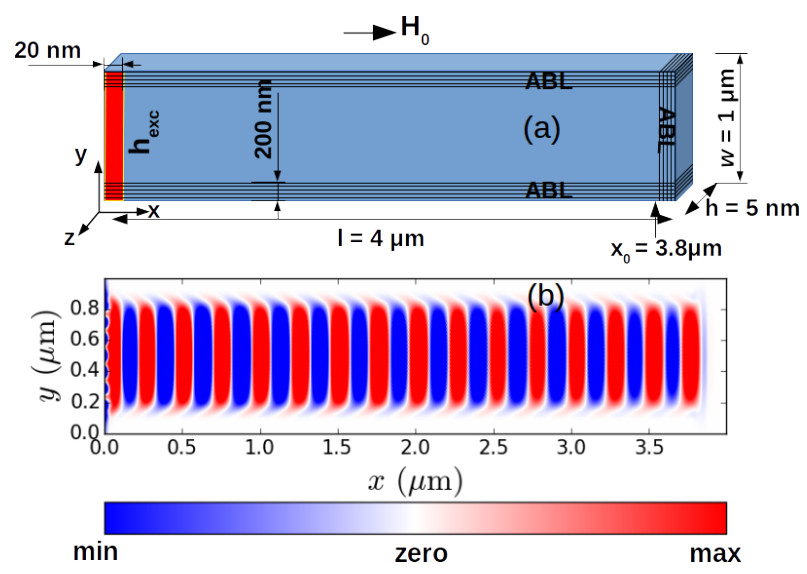

Fig. 1. (a) A magnonic waveguide with absorbing boundary layers along all edges. A SW excitation pulse $\mathbf{h}_{\text {exc }}(t)$, is applied along $\hat{\boldsymbol{y}}$, at the left edge. The origin is at the bottom left corner. (b) A snapshot of $m_{y}$ at $t=500 \mathrm{~ns}$. The colorbar is in linear scale.

A harmonic field excites SWs at the left edge of the stripe so that they propagate along $\hat{\boldsymbol{x}}$. ABLs along the top and bottom stripe edges confine the SWs to the centre of the stripe, as shown in Figure 1 (b). Now, consider different spatial profiles for damping, defined at the right end as:

- constant and abrupt

$$
\alpha_{\mathrm{a}}(x)= \begin{cases}0 & x<3.8 \mu \mathrm{m} \\ 0.1 & x \geq 3.8 \mu \mathrm{m}\end{cases}
$$

- $\tan$ hyperbolic, with $\Delta \alpha=0.5, x^{\prime}=3.9 \mu \mathrm{m}$ and $\sigma_{x}=40 \mathrm{~nm}$, modified from [10]

$$
\alpha_{\mathrm{b}}(x)= \begin{cases}0 & x<3.8 \mu \mathrm{m} \\ \Delta \alpha\left(1+\tanh \frac{x-x^{\prime}}{\sigma_{x}}\right) & x \geq 3.8 \mu \mathrm{m}\end{cases}
$$

- polynomial, with $x_{0}=3.8 \mu \mathrm{m}$

$$
\alpha_{\mathrm{c}, n}(x)= \begin{cases}0 & x<3.8 \mu \mathrm{m} \\ a\left(x-x_{0}\right)^{n} & x \geq 3.8 \mu \mathrm{m} \quad n=1,2\end{cases}
$$

In each case the constants were chosen to obtain $\alpha=1.0$ at $x=4 \mu \mathrm{m}$, as shown in Figure 2. $\alpha_{\mathrm{c}, 1}$ and $\alpha_{\mathrm{c}, 2}$ are linear and parabolic profiles respectively. We compare different profiles over a constant ABL length of $200 \mathrm{~nm}$. In the following sections, we also show that $200 \mathrm{~nm}$ is sufficient for the energy density to decay by over $15 \mathrm{~dB}$, for all the damping profiles.

\subsection{Simulation procedure}

We apply a high bias magnetic field $\mathbf{H}_{0}=804 \mathrm{kA} / \mathrm{m} \hat{\boldsymbol{x}}$, with an artificially high damping $(\alpha=0.5)$, and allow $\mathbf{m}$ to relax to its ground state. Since the magnetization in the stripe is saturated, we do not have any domain walls or vortices in the stripe.

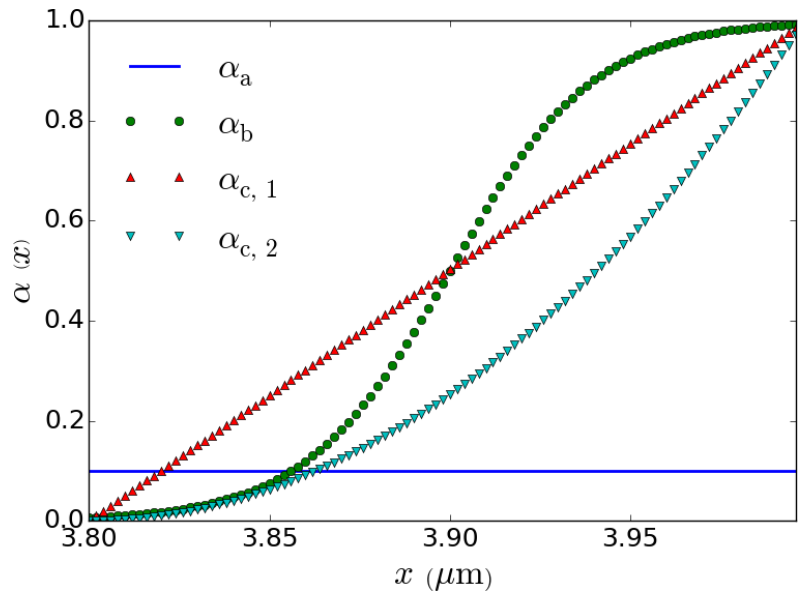

Fig. 2. Spatial variation of different damping profiles that were studied. $\alpha_{c, 2}$ is the parabolic damping profile.

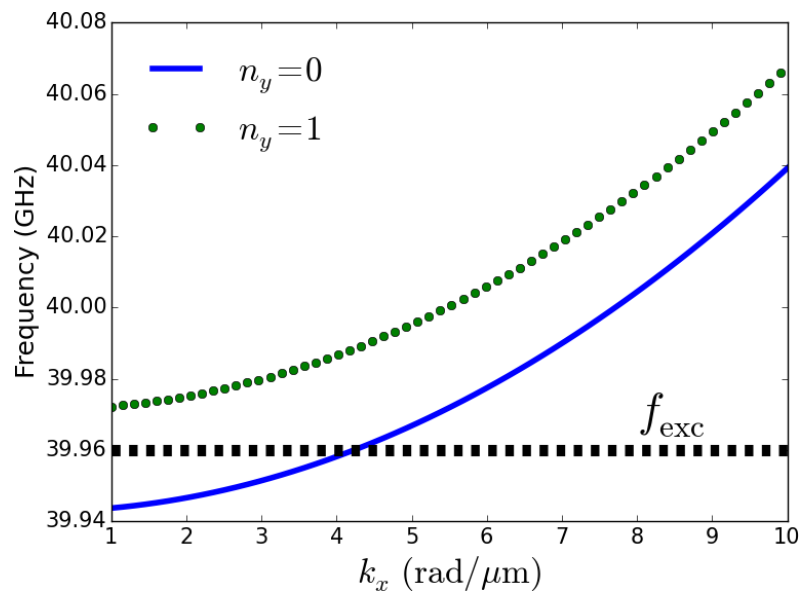

Fig. 3. SW dispersion in the magnonic stripe, showing how $f_{\mathrm{exc}}=39.96 \mathrm{GHz}$ excites only the fundamental mode.

Starting with the ground state, an excitation magnetic field

$\mathbf{h}_{\mathrm{exc}}(x, y, t)=h_{0} \sin \left(2 \pi f_{\mathrm{exc}} t\right) \cos \left(\frac{\pi}{2 w} y-\frac{\pi}{4}\right) \hat{\boldsymbol{y}}$,

is applied at $x<20 \mathrm{~nm}$ (in the region marked in red in Figure 1 (a)) with $h_{0}=0.01 H_{0}, f_{\text {exc }}=39.96 \mathrm{GHz}$ and the width of the stripe $w=1 \mu \mathrm{m}$. A low value of $h_{0}$ ensures that we excite small amplitude SWs. The spatial form of $\cos \left(\frac{\pi}{2 w} y-\frac{\pi}{4}\right)$ was chosen so that we preferentially excite the lowest order width mode.

The dispersion relation for the lowest SW mode in a backward volume geometry ( $\mathbf{k} \| \mathbf{H}_{0}$ ) was derived by Kalinikos [21]. If we include exchange interactions, we get

$$
\begin{aligned}
& \omega=\sqrt{\omega_{\mathrm{ex}}\left(\omega_{\mathrm{ex}}+\omega_{\mathrm{M}} \frac{1-e^{-k h}}{k h}\right)}, \\
& \omega_{\mathrm{ex}}=\omega_{0}+\omega_{\mathrm{M}} \lambda_{\mathrm{ex}} k^{2},
\end{aligned}
$$

where $h$ is the stripe thickness, $\omega_{0}=\gamma \mu_{0} H_{0}$ is the uniform mode precession frequency and $\omega_{\mathrm{M}}=\gamma \mu_{0} M_{\mathrm{S}} . \quad \lambda_{\mathrm{ex}}=\frac{2 A}{\mu_{0} M_{\mathrm{s}}^{2}}$ where $A$ is the exchange constant. $k^{2}=k_{x}^{2}+k_{y}^{2}$ where $k_{x}$ is the propagation constant and $k_{y}=\left(n_{y}+1\right) \frac{\pi}{w}$ is the quantized wave 
vector component along the width. We choose $n_{y}=0$, and pick $f_{\text {exc }}=39.96 \mathrm{GHz}$, to excite only the fundamental mode, as shown in Figure 3. $\mathbf{m}(x, y, z, t)$ is saved at all the nodes of the FD grid. The SWs take approximately $25 \mathrm{~ns}$ to reach the right end of the stripe. We allow the simulation to run till $500 \mathrm{~ns}$ so that the SWs travel ten round trips in the stripe.

\subsection{Transmission line model for ABLs}

The purpose of an ABL is three fold:

1. The SWs should decay sufficiently by the end of the ABL to have no reflections from the stripe edge.

2. The ABL causes minimum reflections back into the device.

3. Minimum energy is reflected into higher order modes.

Consequently, we evaluate the different ABLs, using as a metric the energy density in the ABL and reflections from the ABL.

The energy density of the SWs propagating along the stripe is [17]

$\mathcal{E}(x, y, t)=-\frac{1}{2} \mathbf{M}(x, y, t) . \mathbf{B}(x, y, t)$,

where $\mathbf{B}$ is the instantaneous magnetic flux density. Figure 4 shows the variation of the normalized energy density in the ABL at $t=500 \mathrm{~ns}$. $\mathcal{E}$ decays by over $15 \mathrm{~dB}$ within $200 \mathrm{~nm}$ for all the profiles. We observe no significant reflections from the structure edge, and hence we fix our ABL length at $200 \mathrm{~nm}$ for all the profiles.

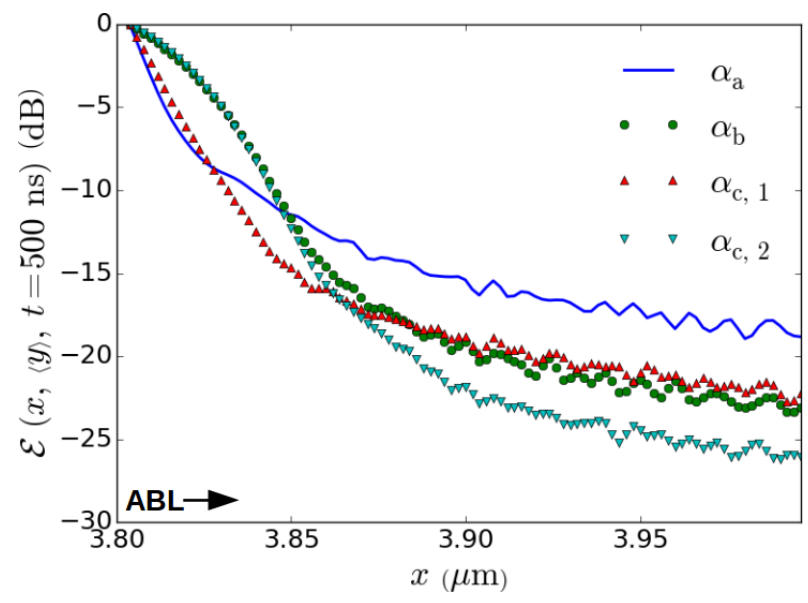

Fig. 4. The decay of the normalized SW energy density $\mathcal{E}$ in the ABL. The energy decays by more than $15 \mathrm{~dB}$ within $200 \mathrm{~nm}$ for all profiles.

We now investigate reflections that originate from the start of the ABL at $x=3.8 \mu \mathrm{m}$. When we make a transition from $\alpha=0$ to $\alpha \neq 0$, we observe SW reflections in a manner analogous to having an impedance mismatch along a transmission (Tx) line. We model the wave propagation in the stripe as standing waves formed on a lossy Tx line. In its simplest form, the magnetization on this line takes the form (c.f. Appendix A)

$m(x)=m^{+}\left[e^{-\zeta x} \cos \beta x+|\Gamma| e^{+\zeta x} \cos (\beta x+\phi)\right]$,

where $m^{+}$is the peak amplitude of the incident wave, $\zeta$ is the loss per unit length, $\beta$ is the propagation constant of the standing wave and $\Gamma=|\Gamma| e^{j \phi}$ is the complex reflection coefficient at the load end. We fit the standing wave $m_{y}(x,\langle y\rangle, t)$, in the stripe, to Eq. (Equation 5) for each of the different damping profiles. These fits are done for $t=475$ to $t=500 \mathrm{~ns}$, to obtain the mean and standard deviation for $\zeta,|\Gamma|$ and $\phi$. One such fit is shown in Figure 5 for $\alpha_{\mathrm{c}, 2}$ at $t_{0}=500 \mathrm{~ns}$.

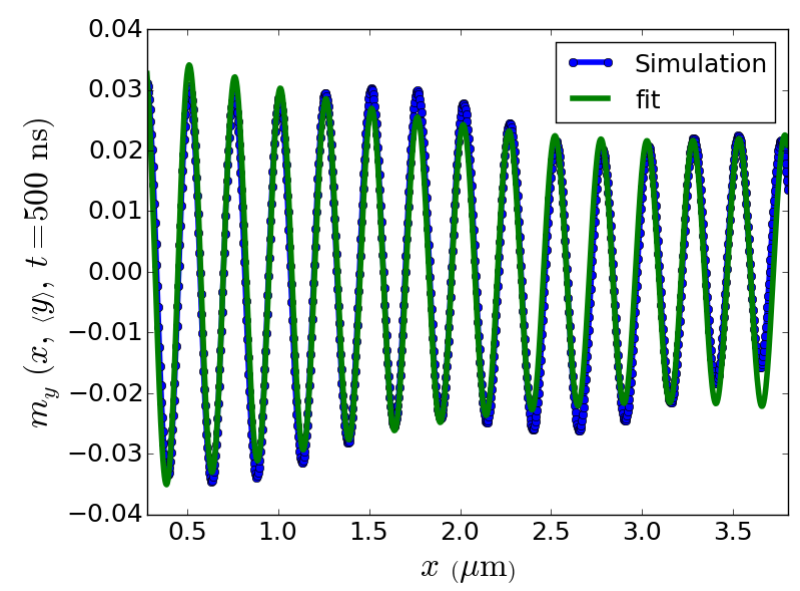

Fig. 5. A fit of Eq. (Equation 5) with the magnetization in the stripe at $t_{0}=$ $500 \mathrm{~ns}$, when the $\alpha_{\mathrm{c}, 2}$ profile is used in the ABL. The fit is used to estimate the return loss appearing in the line due to the introduction of the ABL.

The return loss in a Tx line is a measure of the power reflected by a mismatched load and is given as [22]

$\mathrm{RL}=-20 \log _{10}|\Gamma| \mathrm{dB}$.

The time averaged $\zeta$ and RL values (along with the precision) are given for the different profiles in Table. 1. A higher value of RL indicates a lower reflection coefficient and thus a more matched load, and the parabolic profile shows a $1.5 \mathrm{~dB}$ higher $\mathrm{RL}$ than the commonly used abrupt profile. The value of RL for the parabolic profile $\left(\alpha_{\mathrm{c}, 2}\right)$ is comparable to that of the tan hyperbolic profile $\left(\alpha_{\mathrm{b}}\right)$ and therefore both appear to be efficient for use in an ABL.

Table 1

$\zeta$ and Return loss for the different ABL profiles.

\begin{tabular}{llll}
\hline S. No. & Profile & $\zeta\left(\mu \mathrm{m}^{-1}\right)$ & $\mathrm{RL}(\mathrm{dB})$ \\
\hline 1 & $\alpha_{\mathrm{a}}$ & $0.08 \pm 0.01$ & $5.21 \pm 0.01$ \\
2 & $\alpha_{\mathrm{b}}$ & $0.1 \pm 0.02$ & $6.99 \pm 0.02$ \\
3 & $\alpha_{\mathrm{c}, 1}$ & $0.08 \pm 0.01$ & $5.41 \pm 0.02$ \\
4 & $\alpha_{\mathrm{c}, 2}$ & $0.1 \pm 0.02$ & $6.72 \pm 0.01$ \\
\hline
\end{tabular}

\section{Oblique incidence of spin waves}

In FDTD simulations, the performances of PMLs are typically functions of the angles at which the electromagnetic waves are incident on them. Having shown the performance of ABLs for perpendicular incidence in section 2, we now investigate their effect when we have oblique incidence. Consider the 
geometry recently used to simulate the Goos-Hanchen effect for SWs [23], in a yttrium iron garnet (YIG) film, which is shown in Figure 6. The dimensions of the film are $6000 \times 3000 \times 5 \mathrm{~nm}^{3}$. The material parameters used for YIG were $M_{\mathrm{s}}=194 \mathrm{kA} / \mathrm{m}$ and $A=4 \times 10^{-12} \mathrm{~J} / \mathrm{m}$ [23]. Again no crystalline anisotropy was considered.

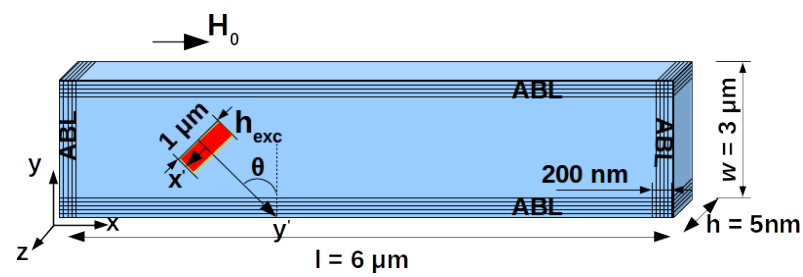

Fig. 6. A thin film of YIG with ABLs all around the periphery and with an excitation region at an angle. The origin is at the bottom left corner.

The $\alpha_{\mathrm{c}, 2}$ profile was applied along the left, top and right edges allowing us to focus on reflections off the bottom edge. We apply a magnetic field $\mathbf{H}_{0}=558 \mathrm{kA} / \mathrm{m} \hat{\boldsymbol{x}}$ and allow $\mathbf{m}$ to relax to its ground state. We then choose an area at angle $\theta$, as shown in Figure 6, and apply [23]

$\mathbf{h}_{\mathrm{exc}}\left(x^{\prime}, y^{\prime}, t\right)=h_{0} e^{-2\left(\frac{x^{\prime}-x_{0}^{\prime}}{l_{\text {exc }} \operatorname{exc}}\right)^{2}} \sin (2 \pi f t) \hat{\boldsymbol{y}}$,

with $h_{0}=0.01 H_{0} . \hat{y}$ is the desired direction of SW propagation, at an angle $\theta$, and $\hat{\boldsymbol{x}}^{\prime}$ is the direction of spin wavefronts. $\left(x_{0}^{\prime}, y_{0}^{\prime}\right)$ marks the centre of the excitation region, and was chosen appropriately for the different angles of incidence considered below, and shown in Fig. 7. The choice of $\left(x_{0}^{\prime}, y_{0}^{\prime}\right)$ ensured that point of incidence was the same for each simulation.

$l_{\mathrm{exc}}=1 \mu \mathrm{m}$ and $w_{\mathrm{exc}}=5 \mathrm{~nm}$ are the length and width of the excitation area, and we apply $\mathbf{h}_{\text {exc }}$ to all mesh nodes that fall within this region. $\sigma_{\text {exc }}=0.4$ decides the spread of the Gaussian envelope. We tested the ABL for sinusoidally pumped spin waves with $f=35 \mathrm{GHz}$ [23].

We observed that proper SW collimation was obtained when the SW propagation angle $(\theta)$ was related to the cell edge lengths, $\Delta x$ and $\Delta y$, by $\tan \theta=\frac{\Delta y}{\Delta x}$. Consequently, we considered three cases where we took $\Delta x=5 \mathrm{~nm}$ and $\Delta y=$ 2.5, 5 and $10 \mathrm{~nm}$. Each of these edge lengths is smaller than the exchange length of YIG $\left(l_{\mathrm{ex}} \approx 13 \mathrm{~nm}\right)$. For these three cases, $\tan \theta=0.5,1$ and 2 which lead to $\theta=26.6^{\circ}, 45^{\circ}$ and $63.4^{\circ}$ respectively.

The snapshots for the $\alpha_{\mathrm{a}}$ and $\alpha_{\mathrm{c}, 2}$ profiles, for the different $\theta$, are shown in Figure 7 . We see significant reflections when $\alpha_{\mathrm{a}}$ is used whereas $\alpha_{\mathrm{c}, 2}$ hardly shows any reflections for the three angles of incidence. The larger reflections from $\alpha_{\mathrm{a}}$ leads to regions of constructive and destructive interference close to the point of incidence. Such artifacts are avoided with $\alpha_{\mathrm{c}, 2}$.

Figure 8 shows the cumulative energy density, from Eq. (Equation 4), in the ABL for the different profiles at $\theta=63.4^{\circ}$. $\alpha_{\mathrm{c}, 2}$ leads to maximum absorption of SWs in the ABL and thus is the most efficient of all the profiles we have considered. Figure 9 shows the magnetization scanned along a wavefront of the reflected wave, which is shown by the red line in Figure 7. Here too the amplitude of the reflected SW beam is low for $\alpha_{\mathrm{c}, 2}$.
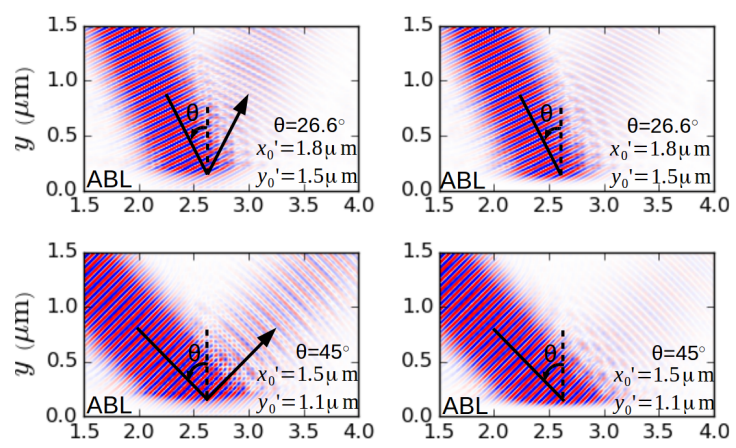

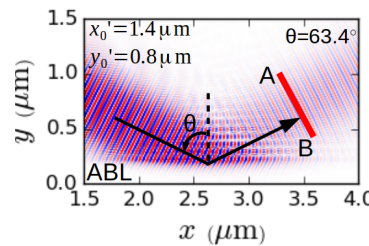

(a)

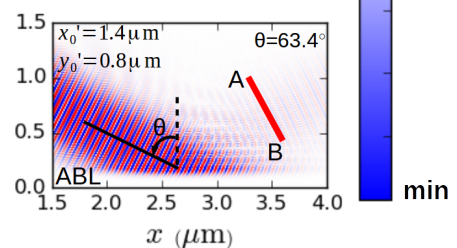

(b)
Fig. 7. SWs in the YIG film for different incident angles. Columns (a) and (b) have the $\alpha_{\mathrm{a}}$ and $\alpha_{\mathrm{c}, 2}$ profiles in the ABL respectively. The colorbar is in linear scale. $\left(x_{0}^{\prime}, y_{0}^{\prime}\right)$ is the centre of the excitation region and was appropriately chosen for each excitation angle. The magnetization is scanned along the wavefront (red line) to obtain the plot in Figure 9. $\alpha_{\mathrm{c}, 2}$ causes minimal reflections for all three angles of incidence.

\section{Summary and conclusions}

Reducing unwanted reflections from boundaries is important for accurate simulations of magnonic devices. Shorter ABLs with abrupt changes in $\alpha$ can cause spurious artifacts. We calculated the return loss introduced in a permalloy stripe due to the SWs normally incident on a ABL, using a transmission line model. The parabolic damping profile yields a higher return loss, $1.5 \mathrm{~dB}$ higher than an abrupt ABL.

We then considered SWs obliquely incident on the ABL at different angles of incidence. Even at a large incidence angle of $63.4^{\circ}$, the parabolic profile $\alpha_{\mathrm{c}, 2}$ causes minimal reflections and leads to the largest SW energy transfer to the ABL. The per-

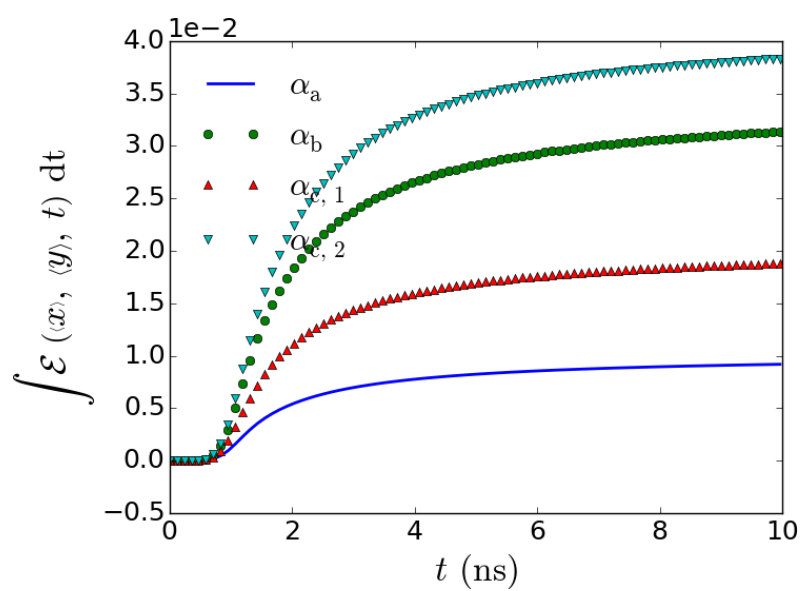

Fig. 8. The cumulative energy density as a function of time in the ABL region for the different profiles at $\theta=63.4^{\circ} . \alpha_{\mathrm{c}, 2}$ shows the largest energy transfer to the ABL. 


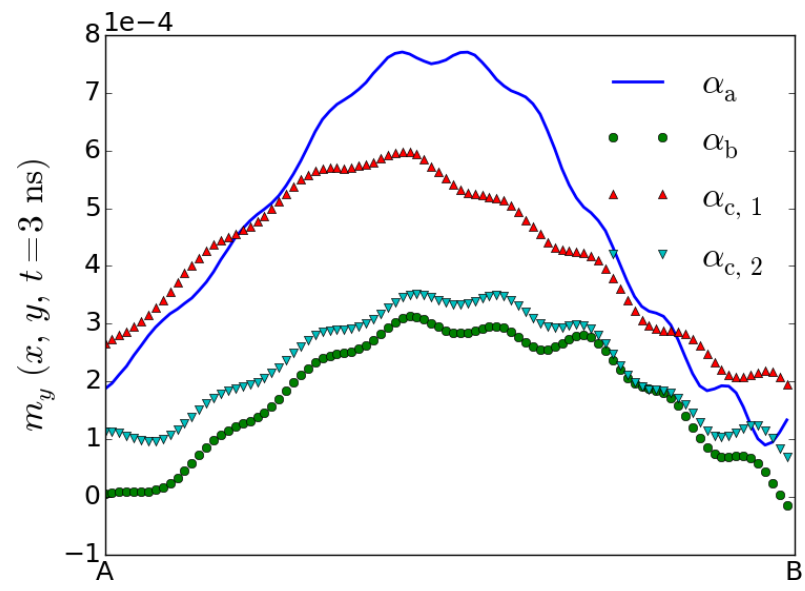

Fig. 9. The variation of the magnetization along the wavefront (red line in Figure 7; simulations were run for $\alpha_{b}$ and $\alpha_{c, 1}$ also). The $\alpha_{\mathrm{b}}$ and $\alpha_{\mathrm{c}, 2}$ profiles show least reflections from the ABL.

formance of the tan hyperbolic damping profile is comparable to that of the parabolic profile. Yet we urge the micromagnetic community to adopt the latter so as to align ourselves with the established use of PMLs in FDTD simulations.

Example scripts to analyze the data, as well as raw data for the figures, are available in the associated electronic supplementary material [18].

\section{Acknowledgments}

The authors would like to thank Malathi M., Manas Srivastava and Rajavardhan $\mathrm{T}$ for fruitful discussions and the High Performance Computing Centre at IIT Madras for the use of their GPU cluster. This work was supported in part by the Department of Science and Technology, Government of India sanction number SB/S3/EECE/011/2014 (IITM).

\section{Appendix A. Standing waves on a transmission line}

Consider a lossy transmission line extending from $x=0$ to $x=x_{0}$. If a wave is launched on a lossy transmission line towards the right at $x=0$ and the line is terminated by an unmatched load, standing waves will be formed on the line at steady state [Pozar 1997]. For small signal magnetization, assuming linear systems, the standing waves are written as a sum of incident and reflected waves as

$m_{\mathrm{tot}}(x)=m^{+} e^{-\zeta x} e^{-j \beta x}+m^{-} e^{+\zeta x} e^{+j \beta x}$,

where $m^{+}, m^{-}, \zeta$ and $\beta$ are the maximum amplitude of incident and reflected waves, the loss per unit length and the propagation constant of the wave respectively. We then have

$m_{\mathrm{tot}}(x)=m^{+}\left[e^{-\zeta x} e^{-j \beta x}+\Gamma e^{+\zeta x} e^{+j \beta x}\right]$,

where $\Gamma=|\Gamma| e^{j \phi}$ is the reflection coefficient at the load end $x=x_{0}$. The real part of $m_{\mathrm{tot}}(x)$ is

$m(x)=m^{+}\left[e^{-\zeta x} \cos \beta x+|\Gamma| e^{+\zeta x} \cos (\beta x+\phi)\right]$.

\section{Appendix B. Implementation of the ABL in Mumax3}

To assist the interested reader, we reproduce the MuMax3 code for setting the parabolic damping profile at the edge of the stripe in Figure 1 (a). We define each cell in the ABL as a region and set the parabolic damping in it. We define the start and stop damping values, and the range of $x$ values.

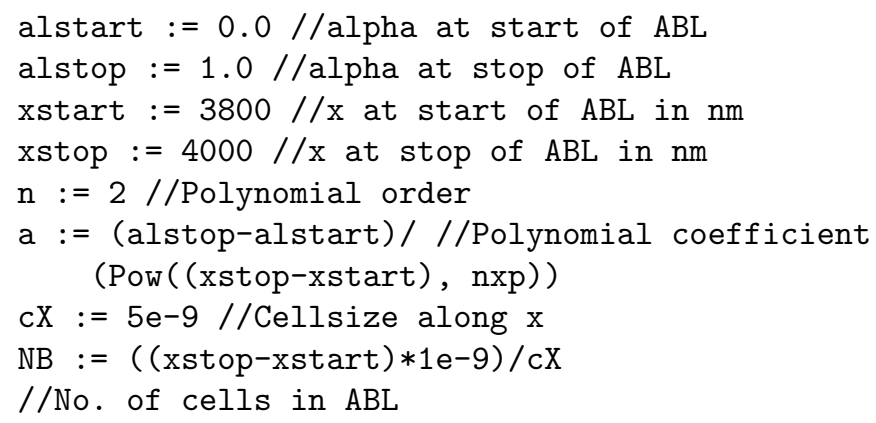

\section{References}

\section{References}

[1] S. Bance, T. Schrefl, G. Hrkac, A. Goncharov, D. A. Allwood, J. Dean, Micromagnetic calculation of spin wave propagation for magnetologic devices, J. Appl. Phys. 103 (7) (2008) 07E735. http://dx.doi.org/ 10.1063/1.2836791.

[2] Y. Peng, G. Zhao, F. Morvan, S. Wu, M. Yue, Dynamic micromagnetic simulation of the magnetic spectrum of permalloy nanodot array with vortex state, J. Magn. Magn. Mater 422 (2017) 57-60. http: //dx.doi.org/10.1016/j.jmmm.2016.08.060.

[3] V. V. Kruglyak, et al., Magnonics, J. Phys. D: App. Phys. 43 (2010) 264001. http://dx.doi.org/10.1088/0022-3727/43/26/ 264001.

[4] S. K. Kim, Micromagnetic computer simulations of spin waves in nanometre-scale patterned magnetic elements, J. Phys. D: App. Phys. 43 (26) (2010) 264004. http://dx.doi.org/10.1088/0022-3727/ 43/26/264004.

[5] Z. Li, M. Wang, Y. Nie, D. Wang, Q. Xia, W. Tang, Z. Zeng, G. Guo, Spin-wave propagation spectrum in magnetization-modulated cylindrical nanowires, J. Magn. Magn. Mater 414 (2016) 49 - 54. http: //dx . doi . org/10.1016/j.jmmm.2016.04.057.

[6] R. Silvani, M. Kostylev, A. Adeyeye, G. Gubbiotti, Spin wave filtering and guiding in Permalloy/iron nanowires, J. Magn. Magn. Mater (2017) -http://dx.doi.org/10.1016/j.jmmm.2017.03.046.

[7] G. Venkat, D. Kumar, M. Franchin, O. Dmytriiev, M. Mruczkiewicz, H. Fangohr, A. Barman, M. Krawczyk, A. Prabhakar, Proposal for a standard micromagnetic problem: Spin wave dispersion in a magnonic waveguide, IEEE Trans. Magn. 49 (1) (2013) 524-529. http://dx . doi .org/10.1109/TMAG . 2012.2206820.

[8] M. Fähnle, A. Slavin, R. Hertel, Role of the sample boundaries in the problem of dissipative magnetization dynamics, J. Magn. Magn. Mater 360 (2014) 126-130. http://dx.doi.org/10.1016/j.jmmm. 2014. 02.031. 
[9] O. Dmytriiev, V. V. Kruglyak, M. Franchin, H. Fangohr, L. Giovannini, F. Montoncello, Role of boundaries in micromagnetic calculations of magnonic spectra of arrays of magnetic nanoelements, Phys. Rev. B 87 (2013) 174422. http://dx.doi.org/10.1103/PhysRevB.87. 174422.

[10] D. V. Berkov, N. L. Gorn, Micromagnetic simulations of the magnetization precession induced by a spin-polarized current in a point-contact geometry (invited), J. Appl. Phys. 99 (8) (2006) 08Q701. http://dx . doi.org/10.1063/1.2151800.

[11] G. Consolo, L. Lopez-Diaz, L. Torres, B. Azzerboni, Boundary conditions for spin-wave absorption based on different site-dependent damping functions, IEEE Trans. Magn. 43 (6) (2007) 2974-2976. http: //dx.doi.org/10.1109/TMAG.2007.893124.

[12] M. Dvornik, A. N. Kuchko, V. V. Kruglyak, Micromagnetic method of s-parameter characterization of magnonic devices, Journal of Applied Physics 109 (7) (2011) 07D350. http: //dx.doi.org/DOI : 10.1063/ 1.3562519.

[13] B. V. de Wiele, F. Montoncello, A continuous excitation approach to determine time-dependent dispersion diagrams in $2 \mathrm{D}$ magnonic crystals, $\mathrm{J}$. Phys. D: App. Phys. 47 (31) (2014) 315002. http://dx . doi .org/10. 1088/0022-3727/47/31/315002.

[14] X. Zhang, M. Ezawa, D. Xiao, G. P. Zhao, Y. Liu, Y. Zhou, Allmagnetic control of skyrmions in nanowires by a spin wave, Nanotechnology 26 (22) (2015) 225701. http: //dx . doi .org/10.1109/int-mag . 2015.7157728.

[15] J. Xia, X. Zhang, M. Yan, W. Zhao, Y. Zhou, Spin-Cherenkov effect in a magnetic nanostrip with interfacial Dzyaloshinskii-Moriya interaction, Scientific Reports 6 (25189). http://dx.doi.org/10.1038/ srep25189.

[16] X. L. Travassos, S. L. Avila, D. Prescott, A. Nicolas, L. Krahenbuhl, Optimal configurations for perfectly matched layers in FDTD simulations, IEEE Trans. Magn. 42 (4) (2006) 563-566. http://dx .doi.org/10 . 1109/TMAG. 2006.871471.

[17] A. Vansteenkiste, J. Leliaert, M. Dvornik, M. Helsen, F. Garcia-Sanchez, B. Van Waeyenberge, The design and verification of MuMax3, AIP Advances 4 (10) (2014) 107133. http://dx.doi.org/10.1063/1. 4899186.

[18] G. Venkat, H. Fangohr, A. Prabhakar, Post processing codes for paper on absorbing boundary layers for spin wave micromagnetics, GitHub. http : //dx.doi.org/10.5281/zenodo. 161326.

[19] L. Landau, L. Lifshitz, Theory of the dispersion of magnetic permeability in ferromagnetic bodies, Phys. Zeit. Sowjetunion 8 (1935) 153. http: //dx.doi.org/10.1016/b978-0-08-010586-4.50023-7.

[20] M. Franchin, Multiphysics simulations of magnetic nanostructures, $\mathrm{Ph} . \mathrm{D}$. thesis, Univ. of Southampton (2009). http: //eprints . soton.ac.uk/ id/eprint/161207

[21] B. Kalinikos, Excitation of propagating spin waves in ferromagnetic films, IEE Proc. 127 (1) (1980) 4. http://dx.doi.org/10.1049/ ip-h-1.1980.0002.

[22] D. Pozar, Microwave Engineering, 2nd Edition, Wiley, 1997.

[23] P. Gruszecki, Y. S. Dadoenkova, N. N. Dadoenkova, I. L. Lyubchanskii, J. Romero-Vivas, K. Y. Guslienko, M. Krawczyk, Influence of magnetic surface anisotropy on spin wave reflection from the edge of ferromagnetic film, Phys. Rev. B 92 (2015) 054427. http://dx.doi.org/10.1103/ physrevb.92.054427. 